\title{
Communicating Intimacy One Bit at a Time
}

\author{
Joseph 'Jofish' Kaye ${ }^{1}$, Mariah K. Levitt ${ }^{2}$, Jeffrey Nevins ${ }^{1}$, Jessica Golden ${ }^{3}$ and Vanessa Schmidt ${ }^{3}$ \\ Cornell University \\ 1:Information Science | 2:Usability Engineering | 3:Communications \\ 301 College Ave, Ithaca NY 14850 \\ jofish|ml233|jbn7|jmg87|vcs3@cornell.edu
}

\begin{abstract}
In this paper, we present a study of 'minimal intimate objects': low bandwidth devices for communicating intimacy for couples in long-distance relationships. We describe a user study of a software intimate object built to communicate a single bit at a time. The results from both log data and journal entries suggest that even a one-bit communication device is seen by users as a valuable and rich channel for communicating intimacy, despite the availability of wider channels of communication such as email, instant messaging, and telephone. We suggest the constrained nature of the communication affords active reinterpretation by its users, and discuss the results in the context of the study of intimacy in human-computer interaction.
\end{abstract}

Keywords: Intimacy, design, intimate technology, gifts, presence, domestic technology, communication.

\section{ACM Classification: H.5.m Miscellaneous}

\section{INTRODUCTION}

HCI has seen an expansion from its roots in military and workplace studies, and has come to recognize the importance of home life and personal relationships [4]. Intimacy is an important element of personal relationships: how can technology play a role in expression and communication of this intimacy? HCI responded to this challenge through the development of devices for communicating intimacy: previous work has included, among many others, elegant designs for physically distant couples to communicate [12] and for communicating from the bed [2]. This work has inspired the need for a focused look at intimacy as a field of research in HCI.

The first meeting centered on intimacy in HCI was the 2003 Intimate Ubiquitous Computing workshop at Ubicomp [1]. Since then, HCI research on intimacy has grounded itself in in-depth studies of real-life relationships, providing a greater understanding of the complexities, opportunities and problems associated with intimacy $[9,10]$.

The key difficulty in researching intimacy is that it is ambiguous, subjective and hard to define [11]. It's holding hands over a candlelit dinner, and yet it's inherent but invisible in mundane discussions about planning vacations and having the in-laws to dinner. What role can

Copyright is held by the author/owner(s).

CHI 2005, April 2-7, 2005, Portland, Oregon, USA.

ACM 1-59593-002-7/05/0004. communication technologies play in the expression or maintenance of this abstract and ethereal connection between people?

\section{Background}

In earlier research we conducted in-depth interviews with four couples in long-distance relationships to build a picture of their current systems for communicating intimacy, and had them sketch novel devices for maintaining a feeling of intimacy at a distance [9]. Kjeldskov et. al. engaged six cohabiting couples in an extended seven-week cultural probe to understand the role of intimacy in their lives [10].

In both of these studies, subjects expressed a desire for novel technology for communicating intimacy by providing presence and activity awareness. Drawing from the results of their cultural probe, Kjeldskov et. al. propose a design they call "Constant Touch", noting that "intimates desired connectedness and presence... a single point transducer (such as a light)...to suggest physical presence as if 'She is with me all the time.”,

What is interesting about such insights is the simplicity of the proposed design, and the concept of meaningful interactions through minimal communication. We were inspired by this proposal to build a technology that could be used to transmit intimacy using the smallest amount of communication possible.

\section{Virtual Intimate Objects}

We built a system that allows a user to send their partner a very simple, one-bit message. Each member of a couple installed our Virtual Intimate Object, or VIO, which appeared as a small circle in the taskbar of the user's Windows screen.

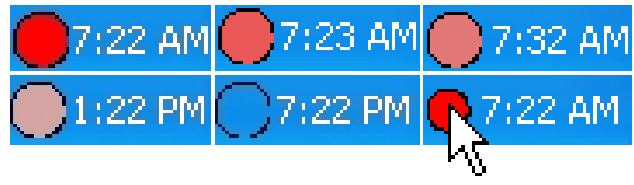

Figure 1: Virtual Intimate Object (VIO) in taskbar, showing color changes over a twelve hour period. Note initial rapid fading in top line. Final image shows display of remote partner's button state on mouseover.

When one member clicks on the circle, their partner's circle changes to bright red. As shown in Figure 1, the circle dims quickly at first, and then fades slowly over time. Eventually, it returns to transparent twelve hours after the circle was clicked. Pressing the button again restarts the cycle at 
maximum light intensity. Moving the mouse over the circle without clicking shows the current status of the remote partner's circle.

\section{METHODS}

\section{Procedure}

We recruited five couples $(\mathrm{n}=10)$ in pre-existing, longdistance romantic relationships and asked them to use the VIO and $\log$ their experiences for a period of one week. Each participant was sent a package by mail that contained instructions, an informed consent form, pre- and post-test questionnaires, and a daily logbook. They were also provided with a pre-stamped and pre-labeled envelope for returning the materials at the end of the study.

The pre-test questionnaire focused on the current modes, frequency, and initiation of communication with their partner. Participants were asked to subjectively rate the effectiveness and level of intimacy of their current methods of communication, and to define their understanding of intimacy in their own words.

Participants were informed that they could choose when and how much to use their VIO during the course of the study, but that it would be available whenever they were using their computers. At the end of each day, they were to reflect on their experience with the device by answering a series of questions in the daily logbook. Some of these questions were the same each day, such as 'How connected did you feel to your partner today?' and 'I think I pressed the button times today. I think my partner pressed the button __times today.' We also included a changing set of open-ended questions, such as 'I generally found I used my VIO when...', 'If my VIO made a sound, it would be...' and 'I would name my VIO...'

Once the couples had used their VIOs for 7 days, they completed the post-test questionnaire. The post-test questionnaire was nearly identical to the pre-test questionnaire, but also sought overall reactions to their VIO, and if the study had any noticeable effect on the participants' level of intimacy with their partner. When all stages of testing were complete, participants mailed back their test materials. Each couple was then thanked for their time, debriefed about the purpose of the experiment, and supplied with their own log data. Couples were also told they were free to continue using their VIOs after the end of the experiment if they so wished.

\section{Tracking}

A message from one VIO is left for the partner's VIO on a central server. The VIOs checked for messages on the server every ten seconds. As such, there was up to a ten second delay between the user clicking the button and seeing the update. The server logs therefore provided data on how often each VIO was clicked, and also the total amount of time each VIO was in use.

In the design of our procedure, we were strongly influenced by technological probes: we wanted to collect information about the use and users of the VIO in a real-world setting, we wanted to field-test the VIO technology, and we wanted to inspire users and designers to think of new kinds of technologies. [5] We feel our design allowed us to explore a wide variety of such issues.

\section{RESULTS}

In the pre-test questionnaire, all couples reported they used telephone, instant messaging and email to stay in touch; some also used text messaging, webcams and paper letters. Our survey results showed that these forms of communication were reported as being only 'somewhat effective' in maintaining intimacy in a long-distance relationship. Participants reported they used their VIO to augment, not replace, these existing channels of communication: "It provided an extra way of connecting to your partner."

On average, couples used their VIOs a total of 35 times a day, although there were wide variations: one couple only used theirs an average of 5 times a day, while another couple clicked the button a total of an average of 123 times a day. All but one of our subjects reported that using their VIO had become part of their daily routine. Seven of the ten participants reported that their VIO made them feel closer to their partner at some point in the study. One respondent wrote, "When I was really busy and couldn't take time to talk or he couldn't - I knew he was still thinking of me when it would go red."

One of the richest parts of our results were the answers to the open-ended questions, which were patterned after cultural probes [7] to try and get an 'impressionistic account' of our subjects' feelings about their relationship and about their intimate object. We asked subjects to reflect on, among other issues, aspects of their relationship, the VIO, and the study itself.

For example, names proposed for the VIOs included "Flipper", "Zit”, "Little Dumbo" and "Bethie's Love". We asked the subjects to name us, the people conducting the research ("Intimacy Dream Team", "Match sustainers (like matchmakers)", "Mysterious Watchers") and to write what they thought the research was really about ("People in relationships trying to connect throughout the day without using or needing words", "Can a simple computer program enhance a long-distance relationship?”, "Creating computer dependency and spreading and marketing it to the general public.”)

These answers are hard to interpret in a traditional manner, but we found that the qualitative, impressionistic answers gave context and explanations for patterns we saw in individual quantitative data. For example, 7 of our 10 respondents said the season that most represented their relationship was spring, while one subject reported summer. Interestingly, the one couple who used their VIO the least of all reported fall and winter as their answer to that question. The point is not that this is a statistically significant result. What we do suggest is that there's a richer picture given by 
this combination of qualitative and quantitative data than we'd get from standard survey techniques.

Finally, one user in an extremely long-distance relationship, spanning ten time zones, wrote to us on the last day of the study. He asked, "Must we uninstall it? I am asking these things, because my girlfriend and I enjoyed a lot using it and I wanted to know if we can...'keep using it' after this study..." In fact, perhaps the strongest indication that couples found their VIOs effective in communicating intimacy is that three of the five couples - six of our ten subjects - continued to use their VIOs after the study ended.

\section{DISCUSSION}

\section{Gifts}

We found the notion of 'gifts' useful in understanding the experience of the VIO. Taylor \& Harper showed how text messages function as gifts between teenagers, and how the social practice of text messaging resembles the anthropological concept of the gift economy [13]. They use the term gift to reference "age-old practices that peoples ceremoniously perform to establish and cement allegiances, and sustain rivalries.” We suggest that each click of the VIO functioned as a gift.

Taylor \& Harper state that the value of a gift is dependent on who sent it, and the symbolic message intended for the recipient. Couples decided for themselves the meaning of each individual click. We found these meanings changed depending on the current situation of each of the participants. A click first thing in the morning could mean "Are you awake?", while a click half an hour after signing off an instant message session to do work could mean "I'm thinking of you". Reciprocating clicks could mean "Yes, call me!" or "I'm thinking of you too." We suggest the feeling of intimacy comes from participation in this cycle of giving and receiving gifts. The process of exchange itself becomes a token of commitment in the relationship.

The obligation of reciprocity in a gift economy also establishes a fertile environment for competition: one of our subjects observed, “...it is a contest to keep up with each other, click for click." Taylor \& Harper suggest that a person who gives a gift is escalated to a position of superiority until the recipient reciprocates the action of giving, thus reestablishing equal status. The lack or delay of reciprocity can greatly diminish the feeling of trust and connectedness from the perspective of the giver. The recipient then feels obligated to reciprocate in the future to prove their commitment to the mutual exchange.

Consistent with this notion, half our respondents mentioned they felt that using the VIO was an obligation, not an optional activity: one subject said, "I would get yelled at by my partner if I didn't press it some days." Similarly, a sizeable portion of our participants also noted the competition that arose from using their VIOs. One participant referred to this competition as "clickwars - who could maintain the bright red.” Another said, “...my partner is bragging about having pushed it more than me and is using it as a game...”

We feel these instances of competition emphasize the situated nature of communication through the VIO, and underline the rich potential for interpretation even in a 1-bit communication device.

\section{Co-Presence and Presence Awareness}

Our users reported that using the VIO gave a sensation of peripheral presence and activity awareness: the knowledge the other person is at their computer and thinking of you, without the interruption of a phone call or instant message. One of our subjects wrote: "I like when it becomes red because I know that [my boyfriend] is clicking on it...and vice versa. It is a nice way to communicate that we are thinking [of] each other."

There is a great deal of work on presence and activity awareness and co-presence in HCI and CSCW. We found the closest parallels to our work in Ito's description of how Japanese mobile phone users in close relationships send text messages back and forth to produce a sense of ambient virtual co-presence, or 'ambient accessibility', defining “a space of peripheral background presence that is midway between direct interaction and non-interaction."[8] The logbooks suggest that the simple knowledge that the other person is also at their computer gives the feeling of copresence: the sensation of sharing a virtual space. As one participant wrote, "[It]give you the sensation that she [is] in front of you, that you can see her pushing the button." Knowing that his girlfriend is at his computer and clicking on his VIO facilitates the sense that they are in a shared space.

\section{LIMITATIONS}

As an initial study of a complicated issue, there are certain limitations to this experiment. Our research looked at only ten people in long-distance relationships. Also, we only asked our subjects to log their experiences using their VIOs for a period of one week: one subject observed "[after] having it for only seven days it was still a novelty; it would be good to use for a month to see what happens." We would like to replicate this study with a much larger sample size, and over a longer period of time.

We explored the concept of minimal intimate objects in the context of the desktop computer. Clearly, there are many other ways to implement a single-bit communication device. Our results may not generalize to intimate objects off the desktop, on platforms such as mobile phones.

\section{CONCLUSION}

Our key finding is that even a one-bit communication device is seen by users as a valuable and rich resource for communicating intimacy, and that it is used despite the availability of wider channels of communication such as email, instant messaging, and telephone. The users interpret the single bit in a wide variety of ways, depending on their individual and joint situations. 
This simple red circle is experienced in many ways: as a gift, as a site for friendly competition, as an informative tool for presence and activity awareness, and as a medium for establishing co-presence. We suggest that this surprising richness of experiences comes from the very simplicity of the VIO: its inherent ambiguity affords active reinterpretation from its users.

\section{FUTURE WORK}

We feel this has been a useful pilot study to understand the potential of minimal intimate objects. However, there are several changes we would like to make in both the study and the intimate objects in the next phase of work. In particular, we would like to do a similar study with a larger number of subjects over a longer period of time.

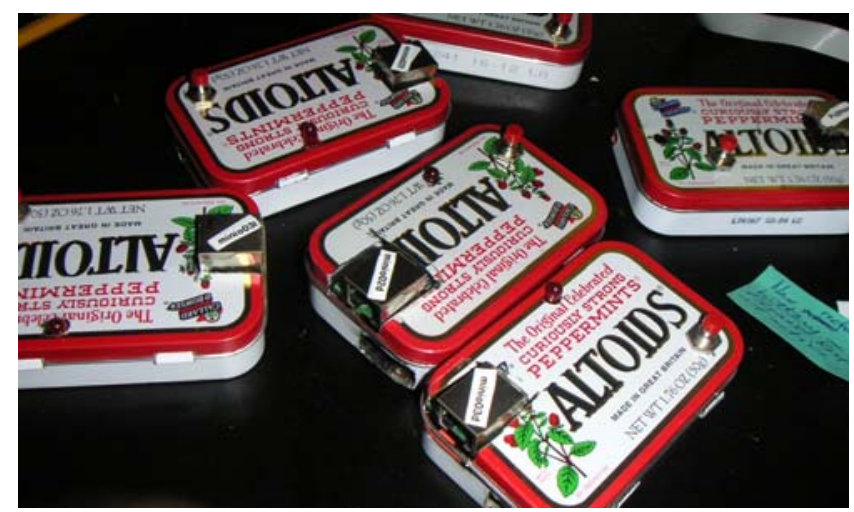

Figure 2: Physical Minimal Intimate Objects

We have recently built physical versions of Minimal Intimate Objects: a box containing a single-board networked computer, a button and an LED, with identical functionality to the VIO. We intend to perform a similar user study using these physical intimate objects and explore the differences in use and satisfaction between the physical and virtual intimate objects.

We are also in the process of releasing the minimal intimate objects client, server software and even logbook as an open source project, to serve as a very low overhead platform for developing similar technological devices, and hopefully exploring IO usage patterns with a large subject pool.

\section{ACKNOWLEDGMENTS}

We would like to thank Jeff Hancock and Kirsten Boehner for their invaluable and repeated assistance. We also had excellent advice from Phoebe Sengers, Michael Lynch, Lucy Suchman and Bill Gaver. This project was funded in part by National Science Foundation grant 0238132 awarded to Phoebe Sengers and by a Hatch grant awarded to Jeffrey $\mathrm{T}$. Hancock.

\section{REFERENCES}

1. Bell, G., Brooke, T., Churchill, E., and Paulos, E., (eds). (2003) Full Workshop Proceedings: Intimate Ubiquitous Computing, Ubicomp 2003.

2. Dodge, C. The Bed: A Medium for Intimate Communication. In Extended Proceedings of CHI'97. (1997) ACM Press.

3. Daft, RL, \& Lengel, RH. Organizational information requirements, media richness and structural design. Management Science, 32, (1986), 554-571.

4. Hindus, D. (1999) The Importance of Homes in Technology Research. Second International Workshop on Cooperative Buildings (CoBUILD '99). Pittsburg PA, Springer.

5. van der Hoog, W., Keller, I., Stappers, P.J.: Gustbowl: technology supporting affective communication through routine ritual interactions. CHI'04 Extended Abstracts (2004) 775-776.

6. Hutchinson, H., W. Mackay, B. Westerlund, B. Bederson, A. Druin, C. Plaisant, M. Beaudouin-Lafon, S. Conversy, H. Evans, H. Hansen, N. Roussel, B. Eiderbck, S. Lindquist, and Y. Sundblad (2003) Technology Probes: Inspiring design for and with familes. In Proceedings of CHI'03. ACM Press. (2003)

7. Gaver, B., Dunne, T., Pacenti, E. Cultural Probes. interactions, 6(1) 21-29 (1999).

8. Ito, M. and Okabe, D. Technosocial Situations: Emergent Structurings of Mobile Email Use. in Personal, Portable Intimate: Mobile Phones in Japanese Life. M. Ito, M. Matsuda, D. Oakabe, (eds.). MIT Press, Cambridge MA, 2005.

9. Kaye, J.'J.' and Goulding, L. Intimate Objects. Proceedings of DIS'04 (2004) ACM Press.

10. Kjeldskov, J., Gibbs, M.R., Vetere, F., Howard, S., Pedell, S., Mecoles, K., Bunyan, M.. Using Cultural Probes to Explore Mediated Intimacy. Proceedings of $\mathrm{OzCHI}$ (2004).

11. Moss, B.F. and A.I. Schwebel. Defining Intimacy in Romantic Relationships. Family Relations. 42,1 (1993).

12. Strong, R. and Gaver, W. Feather, Scent and Shaker: Supporting Simple Intimacy in Videos. In Proceedings of CSCW '96, ACM Press (1996), 29-30.

13. Taylor, A.S., and Harper, R. Age-old practices in the 'New World': A study of gift-giving between teenage mobile phone users. Proceedings of CHI '02, (2002) ACM Press. 